\title{
GFRA3 promoter methylation may be associated with decreased postoperative survival in gastric cancer
}

Lars Lohne Eftang ${ }^{1,2^{*}}$ (D), Jovana Klajic ${ }^{1,3,4}$, Vessela N. Kristensen ${ }^{1,4}$, Jörg Tost ${ }^{5}$, Qin Ying Esbensen ${ }^{1}$, Gustav Peter Blom ${ }^{6}$, Ida Rashida Khan Bukholm ${ }^{7}$ and Geir Bukholm

\begin{abstract}
Background: A large number of epigenetic alterations has been found to be implicated in the etiology of gastric cancer. We have studied the DNA methylation status of 27500 gene promoter regions in 24 gastric adenocarcinomas from a Norwegian cohort, and aimed at identifying the hypermethylated regions. We have compared our findings to the gene expression in the same tissue, and linked our results to prognosis and survival.

Methods: Biopsies from gastric adenocarcinomas and adjacent normal gastric mucosa were obtained from 24 patients following surgical resection of the tumor. Genome-wide DNA methylation profiling of the tumor and matched non-cancerous mucosa was performed. The results were compared to whole transcriptome cDNA microarray analysis of the same material.

Results: Most of the gene promoter regions in both types of tissue showed a low degree of methylation, however there was a small, but significant hypermethylation of the tumors. Hierarchical clustering showed separate grouping of the tumor and normal tissue. Hypermethylation of the promoter region of the GFRA3 gene showed a strong correlation to post-operative survival and several of the clinicopathological parameters, however no difference was found between the two main histological types of gastric cancer. There was only a modest correlation between the DNA methylation status and gene expression.

Conclusions: The different DNA methylation clusters of the tumors and normal tissue indicate that aberrant DNA methylation is a distinct feature of gastric cancer, although there is little difference in the overall, and low, methylation levels between the two tissue types. The GFRA3 promoter region showed marked hypermethylation in almost all tumors, and its correlation with survival and other clinicopathological parameters may have important prognostic significance.
\end{abstract}

Keywords: Gastric cancer, DNA methylation, Gene expression, GFRA3, Survival, Prognosis

\section{Background}

Gastric cancer (GC) is second to lung cancer in worldwide cancer-related deaths, and is the result of a complex interplay between chronic Helicobacter pylori infection, human genetic factors and environmental carcinogens. However, it is becoming increasingly clear that cancer development is as much a result of epigenetic

\footnotetext{
* Correspondence: lars.eftang@medisin.uio.no

'Department of Clinical Molecular Biology and Laboratory Science (EpiGen), Akershus University Hospital, Division of Medicine, Lørenskog, Norway

${ }^{2}$ Department of Gastrointestinal Surgery, Akershus University Hospital, N-1478 Nordbyhagen, Lørenskog, Norway

Full list of author information is available at the end of the article
}

alterations, as it is a genetic disease [1]. Genetic mutations are relatively infrequent in $\mathrm{GC}$, whereas epigenetic alterations such as DNA methylation may be much more important in promoting GC [2]. DNA methylation is an heritable modification of gene activity, which does not make alterations to the DNA sequence, but involves attachment of a methyl group to the carbon 5 position of cytosines, most commonly where cytosine occurs next to guanine, separated by phosphate known as a CpG dinucleotide. Most focus has been directed at DNA hypermethylation, however whole genome hypomethylation is prevalent in several cancers [3-5]. Hypermethylation of 
gene promoter regions may result in silencing of tumor suppressor genes, however the role of hypomethylation has been less clear, but may be associated with increased expression of oncogenes [6]. A distinctive DNA methylation phenotype has been identified in colorectal cancer (CIMP) [7], and particular methylation patterns have been associated with subgroups of breast cancer [8], lung cancer [9] and glioma [10]. A CIMP in gastric cancer has been suggested, but is controversial [11]. Several causes of aberrant DNA methylation in GC have been established, including aging, dietary causes and microorganisms such as H. pylori and Ebstein-Barr virus [12-16].

In a previous study we compared the gene expression profile of 20 gastric tumors against matched noncancerous mucosa. We identified the most differentially expressed genes and related these to postoperative survival [17]. Nine genes relevant to gastric carcinogenesis had previously shown similar expression patterns in $H$. pylori exposed gastric mucosa cells in vitro [18]. We suggested that the increased expression of these genes in the gastric tumors may represent early events in gastric carcinogenesis mediated by chronic $H$. pylori infection, in particular claudin-1 (CLDN1) and interleukin-8 (IL-8).

The aim of the present study was to examine the DNA methylation status in gastric adenocarcinomas whose gene expression were previously determined by cDNA microarrays [17]. We aimed at assessing the overall state of hypo- or hypermethylation in the tumors, and identify whether there was an association between the DNA methylation status, clinicopathological factors and gene expression.

\section{Methods}

\section{Tissue and patient characteristics}

Patients with non-cardia gastric adenocarcinoma were identified during upper endoscopy at the outpatient clinic at Akershus University Hospital, Norway, with a histopathological diagnosis of diffuse or intestinal type GC. Thoraco-abdominal computed tomography imaging was performed to exclude patients with distant metastatic disease, ineligible for curative surgery. On admission for surgery, written, informed consent was obtained from all participants in the study. Immediately following the removal of the principal surgical specimen, two tissue samples from each patient were obtained: one from the tumor mass border and another from healthy gastric corpal mucosa, at least $5 \mathrm{~cm}$ away from the tumor. The samples were immediately fresh frozen on dry ice, before definitive storage at $-80{ }^{\circ} \mathrm{C}$. All sample acquisition and handling were performed by the same individual. The tumors were verified and further classified by two senior specialist pathologists. The study was approved by the Regional Ethics Committee (REK) and the ethics committee at Akershus University Hospital. All samples and patient data were coded and blinded before analysis. Patients, clinicopathological characteristics, diagnostic are presented in Table 1.

\section{Methylation assays}

Total DNA was extracted from the fresh frozen tissue using Dneasy Blood and tissue kit (Qiagen $\mathrm{GmBH}$, Germany) according to the manufacturer's standard preparation protocol. DNA concentration and 260/280 ratio were then assessed using a NanoDrop ND-1000 spectrophotometer (NanoDrop Technologies, USA), and found to be adequate for further analysis. $500 \mathrm{ng}$ of DNA was bisulphite converted using the EpiTect 96 Bisulfite Kit (Qiagen GmbH, Germany). Effective bisulphite conversion was verified by absolute quantification assay using Applied Biosystem7900HT/7900HTFast Real Time PCR System Amplification and pairs of primers specific for either converted or unconverted DNA. An aliquot of $4 \mu \mathrm{L}$ of the bisulphite converted DNA was used to perform genome-wide DNA methylation profiling of 24 gastric tumors against matched non-cancerous mucosa using the Illumina HumanMethylation27 BeadChip. This platform detects the methylation status of 27 578 different CpG sites in $>14000$ promoters in the human genome. The experiment was performed using the

Table 1 Patient characteristics and clinicopathological features of the 24 gastric tumors used in the study

\begin{tabular}{|c|c|c|}
\hline \multicolumn{2}{|c|}{ Number and sex of patients } & $\begin{array}{l}n=24 \text { (females } n=7 \\
\text { males } n=17 \text { ) }\end{array}$ \\
\hline \multicolumn{2}{|l|}{ Ethnicity } & Caucasian $n=21$ Asian $n=3$ \\
\hline \multirow{3}{*}{\multicolumn{2}{|c|}{ Age at surgery }} & Total: 68.4 years $( \pm 12.3)$ \\
\hline & & Females: 65.4 years $( \pm 21.5)$ \\
\hline & & Males: 69.5 years $( \pm 8.5)$ \\
\hline \multicolumn{2}{|c|}{$\begin{array}{l}\text { Postoperative survival } \\
\text { (deceased individuals) }\end{array}$} & 13.3 months $( \pm 8.8)$ \\
\hline \multicolumn{2}{|c|}{$\begin{array}{l}\text { Postoperative survival } \\
\text { (alive individuals at study end) }\end{array}$} & 46.0 months $( \pm 8.0)$ \\
\hline \multicolumn{2}{|l|}{ Tumor size } & 47 mm ( \pm 28$)$ \\
\hline \multirow[t]{4}{*}{ Tumor stage } & $\mathrm{T} 1$ & 3 \\
\hline & $\mathrm{T} 2$ & 13 \\
\hline & T3 & 5 \\
\hline & T4 & 3 \\
\hline \multirow[t]{4}{*}{ Nodal stage } & No & 11 \\
\hline & N1 & 8 \\
\hline & N2 & 3 \\
\hline & N3 & 2 \\
\hline \multirow[t]{3}{*}{ Histological type } & Intestinal & 6 \\
\hline & Diffuse & 14 \\
\hline & Mixed & 4 \\
\hline
\end{tabular}

Values are the mean plus/minus standard deviation where appropriate. The details of gender associated with death and survival have been combined 
Infinium Methylation Assay Experienced User Card protocol. All steps were performed according to the Infinium protocol.

\section{Immunohistochemistry}

The presence of $H$. pylori in the surgical specimens was analyzed using a polyclonal anti-Helicobacter-antibody (Dako, Denmark, code B0471, dilution 1:200). $4 \mu \mathrm{m}$ sections of formalin-fixed, paraffin-embedded tissue from non-tumorous mucosa were applied on coated slides. Deparaffinization, rehydration and epitope retrieval were performed in a Dako PT Link (Dako, Denmark) at $97{ }^{\circ} \mathrm{C}$ for $20 \mathrm{~min}$. The immunostaining procedure was carried out in a Dako Autostainer Plus applying the Envision ${ }^{\mathrm{Tw}}$ Flex, High pH system (Dako, Denmark).

\section{Statistical analysis}

Data was processed using the lumi R package. All probes that contained a "zero" value in at least one sample for methylated and unmethylated signals were removed from further analysis. Intra-sample normalization consisted of color bias correction, which is the normalization between the two color channels, and background level correction, using the negative control probes present on the array. Lastly, quantile normalization was performed on the intensities of methylated and unmethylated probes separately, instead of the summarized methylation levels. $\beta$ values (the degree of methylation) were used for further analysis. The $\beta$-value at each CpG site represents continuous value from 0 to 1 where 0 is fully unmethylated and 1 is entirely methylated at that locus. The $\Delta \beta$ value is the difference between the $\beta$ value of the tumor sample and that of the normal sample, ranging from -1 to 1 .

The $\Delta \beta$ value was calculated for all CpG sites in all sample pairs, and the data were loaded into the Jexpress software package [19]. Rank product testing [20] was then performed to test whether the differential methylation between tumor tissue and matched normal mucosa was significant. The $\Delta \beta$ value was declared significant if the adjusted p-value, i.e. the FDR q-value, was less than 0.05. Hierarchical clustering was performed using utilizing the J-express software package [19].

The filtered dataset, consisting of the 200 most significant $\Delta \beta$ values, was imported into Pathway Express, part of the Onto-Tools software suite [21, 22], for KEGG (Kyoto Encyclopedia of Genes and Genomes) cellular signaling pathway analysis [23]. Pathway Express calculates an Impact Factor (IF), which is used to rank the affected signaling pathways, based on the fold change, the number of the involved genes in the pathway, and the amount of perturbation of downstream genes [24].

The filtered dataset was then entered into SPSS Statistics (IBM Software, New York, USA, version 22.0.0.1) to perform correlation analysis to select differentially methylated gene promoter regions that associated with gene expression and clinicopathological parameters. Both Pearson and Spearman correlation coefficients were employed to identify associations. In the one $\mathrm{CpG}$ site that was highly correlated to survival, different cutoff levels were applied to construct high and low methylated groups, before statistical significance between the groups was assessed using a log-rank (Mantel-Cox) test. A Kaplan-Meier survival plot was created to demonstrate the difference in survival between the high and low expression groups. Linear regression analysis was then performed, to predict determinants of CLDN1 expression, using Stata (StataCorp LP, Texas, USA, version 13.1).

The DNA methylation data are available in the ArrayExpress database under the accession number EMTAB-3813 (http://www.ebi.ac.uk/arrayexpress/experiments/E-MTAB-3813).

\section{Results \\ General description}

To investigate the DNA methylation status of $24 \mathrm{GC}$ samples and matched controls, more than 27500 gene promoter sites were examined using the Illumina Infinium HumanMethylation27K platform. First, the methylation status of the entire dataset was considered. Most CpG sites, including both the tumor and normal tissue, demonstrated low levels of methylation, i.e. low $\beta$ values. Second, there was little variance between the samples within each locus, even between the tumor and normal groups. This is illustrated in the histogram in Fig. 1 and in the colored heatmap in Fig. 2.

Then, the $\Delta \beta$ values for each CpG site were calculated. $53.1 \%$ of all sites demonstrated a net positive value, whereas $46.9 \%$ demonstrated a net negative value, indicating a slight overall increase in $\mathrm{CpG}$ methylation in the tumors.

\section{Rank product testing}

Of the $27500 \mathrm{CpG}$ sites on the array, 1660 CpG sites, corresponding to 1194 genes, showed statistically significant increased methylation in the tumor relative to normal mucosa, whereas $1276 \mathrm{CpG}$ sites, corresponding to 1017 genes, showed significant decreased methylation in the tumor relative to normal mucosa, supporting the trend from the entire dataset.

The dataset of all significant CpG sites was then subjected to hierarchical clustering using average linkage and Euclidean distance measure, using the J-express 2012 software package [19]. There was a tendency for the tumor samples to cluster together, and for the normal samples to cluster together, indicating stronger methylation similarities within each of the two groups, than within each of the sample pairs (Fig. 3). 


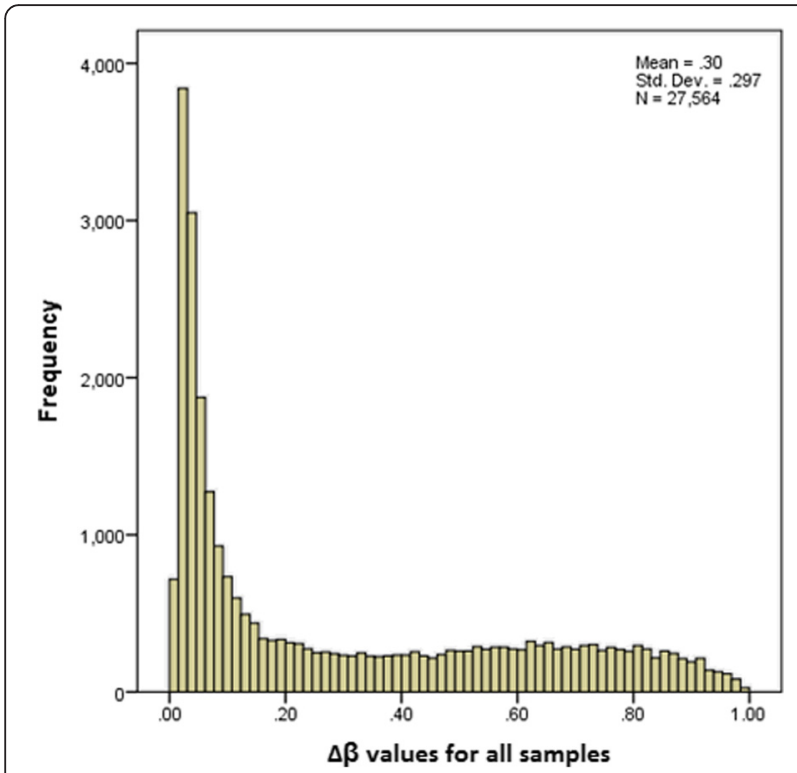

Fig. 1 Histogram of the $\Delta \beta$ values. The frequency of the $\Delta \beta$ values in the dataset of $>27000 \mathrm{CpG}$ sites shows that most $\mathrm{CpG}$ sites demonstrated little variance between gastric tumor and normal tissue

\section{Filtering of statistical CpG sites}

To produce a reasonably sized list of the most differentially methylated CpG sites, a list of the 100 most hypermethylated and the 100 most hypomethylated CpG sites in the tumor relative to the control were created, hereby referred to as the filtered dataset (Additional file 1).

\section{Clinicopathological correlation}

The filtered dataset was then compared to post-operative survival, tumor size, Lauren classification, lymph node metastasis, gastric mucosa metaplasia and atrophy and $H$. pylori, listed in Table 2.

Although 44 CpG sites showed relationship with one or more of the factors, the promoter region of the GFRA3 gene showed significant relationship with nearly all the clinicopathological factors. Firstly, an inverse relationship was detected between the GFRA3 promoter $\Delta \beta$ values and post-operative survival $(p=0.01)$. High and low methylated GFRA3 groups were constructed using the GFRA3 promoter $\Delta \beta$ mean $(p=0.017)$ as the group divider, as demonstrated in the Kaplan-Meier survival plot in Fig. 4. Secondly, a strong, and highly statistically

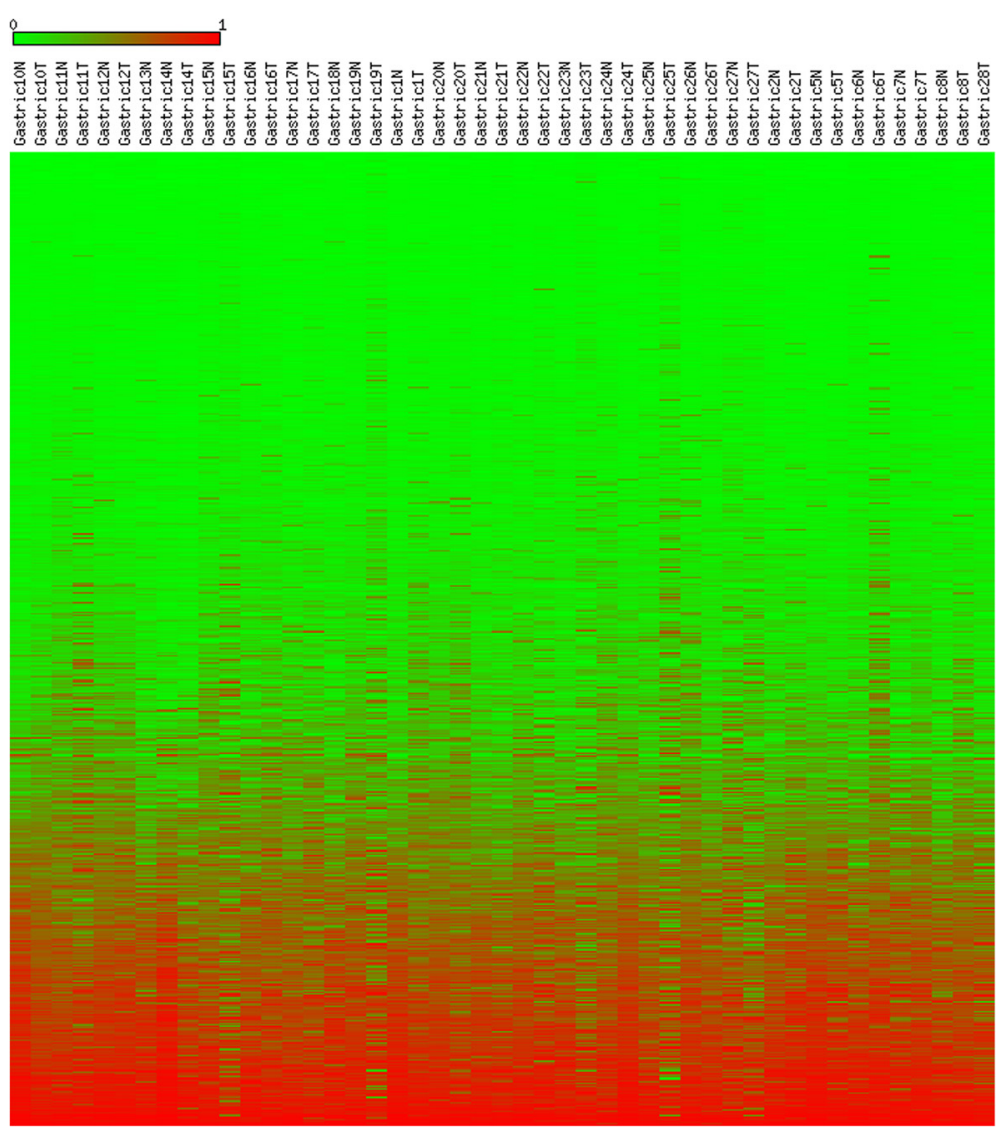

Fig. 2 A heatmap presentation of the DNA methylation levels of the entire dataset of $27564 \mathrm{CpG}$ sites in 24 tumor and normal gastric cancer tissue pairs. A value of 0.00 (most green) indicates fully unmethylated, whereas a value of 1.00 (most red) indicates entirely methylated locus. The figure demonstrates that the majority of $\mathrm{CpG}$ sites in the dataset, including both tumor and normal tissue, showed low levels of DNA methylation 


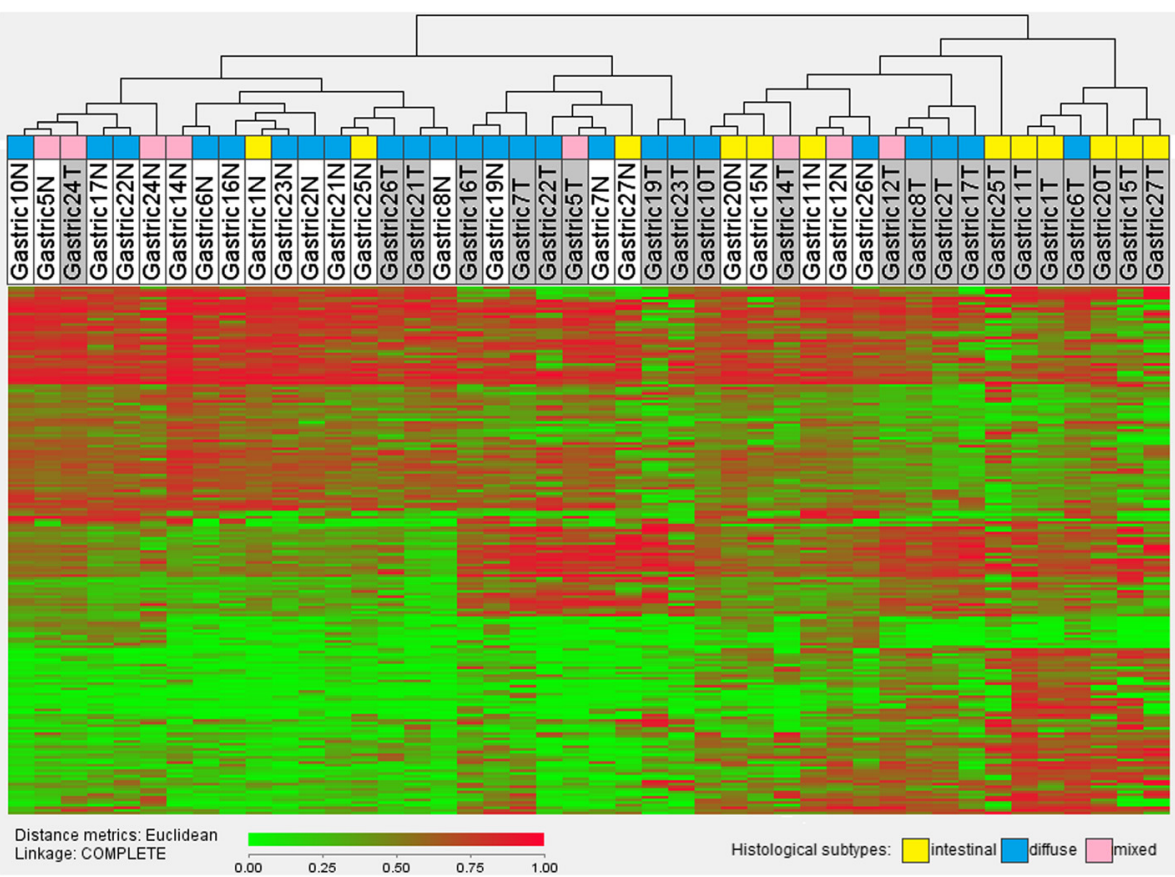

Fig. 3 Hierarchical clustering of the normal and tumor samples. Top row: Tumor samples are shaded grey, normal gastric tissue samples are white. A value of 0.00 (most green) indicates fully unmethylated, whereas a value of 1.00 (most red) indicates entirely methylated locus. Most normal tissue samples seem to concentrate on the left side of the heatmap, whereas most tumor samples aggregate on the right, indicating similarities within the two groups

significant positive relationship was identified between GFRA3 $\Delta \beta$ and gastric atrophy $(p<0.001)$. Thirdly, there were statistically significant, although slightly weaker positive correlations between GFRA3, $\triangle \beta$ and lymph node metastasis $(p=0.028)$, the degree of gastric mucosa metaplasia (0.044) and also patient age at surgery $(p=$ 0.038). There was no association between the GFRA3 promoter methylation levels and histological subtype or H. pylori status, nor did we find significant correlation to GFRA3 gene expression extracted from our previous publication [17].

A multivariable Cox regression was then performed. Controlling for age at surgery, tumor size and histological type, GFRA3 methylation level was still a highly significant $(p=0.01)$ predictor of survival.

\section{Gene expression correlation}

The filtered dataset was compared to corresponding gene regulation, imported from our previous study [17], to analyze whether the gene promoter methylation status could account for aberrant gene expression. Of the 100 most hypermethylated $\mathrm{CpG}$ sites in the tumor relative to the control, four CpG sites showed a statistically significant inverse correlation with gene expression, listed in Table 3. Among the 100 most hypomethylated CpGs, six CpG sites showed significant inverse relationship with gene expression (Table 4).
The 130 most differentially regulated genes in the tumors [17] were then compared against DNA methylation (Table 5). Three genes showed an inverse relationship with the methylation status of their gene promoter region.

Then, to investigate the association between the most significant genes from our previous study [17], and GFRA3 gene methylation from the current study, linear step-wise regression was performed. $I L-8$ gene expression $(p=0.006)$ and GFRA3 methylation $(p=0.008)$ were highly associated with CLDN1 expression.

\section{Cellular signaling pathways}

Then the filtered dataset was analyzed for associated KEGG signal pathways using Pathway Express. Significantly impacted pathways and corresponding Impact Factor (IF) are presented in Table 6. None of the hypermethylated CpG sites (in the tumor relative to normal) associated with any KEGG pathways, while many of the hypomethylated CpG sites significantly associated with six cellular signaling pathways; including three cancerrelated pathways.

\section{Assorted genes}

Finally, the $\beta$ and $\Delta \beta$ values for particular gene promoter regions were noted: TIMP3, SEMA3B, FBP2, TEAD4, CDH1, CDKN2A, LOX, MLH1, and SFRP1, 2 
Table 2 Associations between hypermethylated and hypomethylated CpG sites and clinicopathological features

\begin{tabular}{|c|c|c|c|c|c|c|c|}
\hline & Post-op survival & Lymph node metastasis & Tumor size & Lauren classification & Metaplasia & Atrophy & H. pylori \\
\hline \multicolumn{8}{|c|}{$\begin{array}{l}\text { CpgID/Gene promoter } \\
\text { Hypermethylated in the tumor }\end{array}$} \\
\hline cg09350274/GFRA3 & $r=-0.69 p=0.002$ & $r=0.52 p=0.028$ & $r=0.48 p=0.42$ & & $r=0.480 p=0.044$ & $r=-0.590 p=0.01$ & \\
\hline cg02720618/ESR1 & $r=0.56 p=0.016$ & & & & & & \\
\hline cg04623837/HCG9 & & & $r=0.64 p=0.004$ & & & & \\
\hline cg07307078/TUBB6 & & & $r=0.49 p=0.04$ & & & & \\
\hline cg08615333/TGFB3 & & & $r=0.5 p=0.037$ & & & & $r=0.504 p=0.033$ \\
\hline cg01566170/CAPN2 & & & & $r=-0.53 p=0.023$ & & $r=0.526 p=0.025$ & \\
\hline cg16986846/SCGB2A1 & & & & $r=-0.576 p=0.012$ & & & \\
\hline cg02633817/FXYD3 & & & & & $r=-0.474 p=0.047$ & & \\
\hline cg20640433/LAMA2 & & & & & $r=524 p=0.026$ & & \\
\hline cg21905630/GSH2 & & & & & $r=0.52 p=0.028$ & & \\
\hline cg13718960/RNASE1 & & & & & & $r=0.554 p=0.017$ & \\
\hline cg19118812/ELMO1 & & & & & & $r=-0.546 p=0.019$ & \\
\hline cg26557658/FAM43B & & & & & & $r=-0.604 p=0.008$ & \\
\hline cg03616357/FLJ21159 & & & & & & & $r=0.574 p=0.013$ \\
\hline cg08615333/TGFB3 & & & & & & & $r=0.504 p=0.033$ \\
\hline cg14189571/ZFP42 & & & & & & & $r=0.484 p=0.042$ \\
\hline cg17872757/FLI1 & & & & & & & $r=0.495 p=0.037$ \\
\hline cg21790626/ZNF154 & & & & & & & $r=0.573 p=0.013$ \\
\hline cg27546237/COL4A1 & & & & & & & $r=0.527 p=0.025$ \\
\hline
\end{tabular}

CpgID/Gene promoter

Hypomethylated in the tumor
cg13694749/SCN4A
cg18059088/HS3ST
$r=-0.474 p=0.047$
$r=-0.638 p=0.004$
cg26619317/CNN3
$r=0.481 p=0.043$
cg07131544/NCR2
$r=0.496 p=0.036$

cg14696870/FCER1A

cg20676475/LCE3D

cg00974864/FCGR3B

cg13180098/RHO

cg24691453/S100A4

cg15309006/LOC63928

$r=-0.567 p=0.014$
$r=-0.708 p=0.001$
$r=-562 p=0.015$
$r=-0.604 p=0.008 \quad r=-0.597 p=0.009 \quad r=0.682 p=0.002$
$r=0.470 p=0.49$
$r=-0.499 p=0.035$


Table 2 Associations between hypermethylated and hypomethylated CpG sites and clinicopathological features (Continued)

\begin{tabular}{|c|c|c|c|c|}
\hline cg26789453/TMEM1 16 & $r=-0.512 p=0.030$ & & & \\
\hline cg26264314/NALP5 & $r=-0.474 p=0.047$ & & & \\
\hline cg20383064/BFSP2 & $r=-0.487 p=0.040$ & & & \\
\hline cg25119415/MNDA & $r=-0.646 p=0.004$ & $r=-0.514 p=0.029$ & $r=0.547 p=0.019$ & \\
\hline cg14603345/BTBD3 & & $p=-0.483 r=0.042$ & & \\
\hline cg17356733/IFNGR2 & & & $r=-0.480 p=0.044$ & \\
\hline cg23756272/BCL2 & & & $r=-0.500 p=0.035$ & \\
\hline cg00842351/TJP2 & & & $r=-527 p=0.24$ & \\
\hline cg25248094/SH2D1A & & & $r=0.487 p=0.041$ & $r=-0.526 p=0.025$ \\
\hline cg04454050/TREML1 & & & & 0.4740 .047 \\
\hline cg02611419/KCNK17 & & & & $r=-502 p=0.034$ \\
\hline cg05252264/FCAR & & & & $r=0.587 p=0.10$ \\
\hline cg22268164/TRHR & & & & $r=-0.619 p=0.006$ \\
\hline cg02046017/LOC220070 & & & & $r=0.475 p=0.046$ \\
\hline cg09191232/PAPSS1 & & & & $r=-0.581 p=0.011$ \\
\hline
\end{tabular}

The filtered list of 100 hypermethylated and 100 hypomethylated CpG sites were compared to associated gene expression. 19 of the hypermethylated CpG sites and 25 of the hypomethylated CpG sites showed significant correlation with one or more of the clinicopathological parameters (Pearson correlation coefficients $(r)$ and significance levels $(p)$ are listed) 


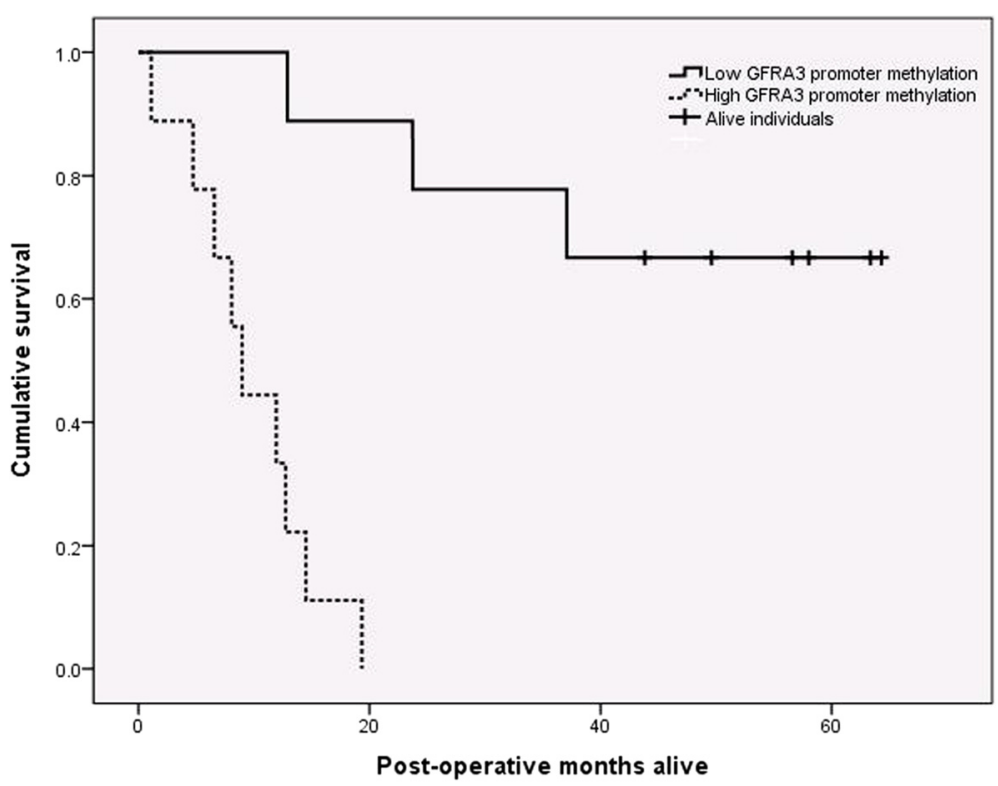

Fig. 4 Kaplan Mayer survival plot of patients with resected gastric tumors. High and low methylated GFRA3 groups were constructed, using the mean value as the group divider. Individuals with hypermethylation of the GFRA3 promoter region showed a highly unfavorable prognosis, whereas individuals with a low degree of methylation at that locus demonstrated a relatively good prognosis

and 5 (Additional file 1), which are addressed in the discussion.

\section{Discussion}

\section{General description}

Although there is currently a major interest in the relationship between DNA methylation and various human diseases, the significance of DNA methylation was highlighted more than 30 years ago by Ehrlich et al. [25], who showed that the extent of methylation varies significantly across human tissues. However, gastric tissue was not studied in detail. In this study we have characterized the DNA methylation pattern in 24 GCs compared to matched normal mucosa. Overall, both tumor and normal mucosa tissue demonstrated similar methylation levels across the genome, where at least two thirds of the $27500 \mathrm{CpG}$ sites showed a very low degree of methylation. The tumor and normal tissues showed very

Table 3 Correlation between hypermethylated gene promoters and corresponding gene expression

\begin{tabular}{lllcl}
\hline $\begin{array}{l}\text { Gene promoter/ } \\
\text { gene }\end{array}$ & $\begin{array}{l}\text { Pearson } \\
\text { coefficient }\end{array}$ & $P$ value & Spearman's rho & $P$-value \\
\hline CPD & & NS & -0.482 & 0.043 \\
DEFB106A & -0.531 & 0.23 & -0.529 & 0.024 \\
FCGR3B & -0.575 & 0.13 & -0.569 & 0.014 \\
GATA4 & & NS & -0.643 & 0.004 \\
\hline
\end{tabular}

The 100 most significant hypermethylated gene promoter sites were correlated with their respective gene expression. Only statistically significant correlations are shown $(p<0.05)$ similar overall methylation patterns, however there was a slight net increase in the global DNA methylation levels in the tumor. This contrasts the belief that global DNA hypomethylation is a general hallmark of all cancer. In concordance with other recent studies we found increased DNA methylation in the tumors in the promoter regions of TIMP3, SEMA3B, FBP2, CDH1, $C D K N 2 A, M L H 1$, and SFRP1,2 and 5 [19-22, 26-31].

Hierarchical clustering of the entire dataset illustrated different methylation patterns between the tumor and the control tissue, and this exercise may distinguish tumor from adjacent tissue with relative accuracy. Statistically significant subclustering between the histological subtypes, according to the Lauren classification, was not seen, probably because of the low sample size. However, the aggregation of the intestinal tumor samples towards

Table 4 Correlation between hypomethylated gene promoters and corresponding gene expression

\begin{tabular}{lllll}
\hline $\begin{array}{l}\text { Gene promoter/ } \\
\text { gene }\end{array}$ & $\begin{array}{l}\text { Pearson } \\
\text { coefficient }\end{array}$ & $P$ value & Spearman's rho & $P$-value \\
\hline DHX32 & -0.495 & 0.037 & -0.624 & 0.006 \\
PPYR & & NS & -0.490 & 0.039 \\
FOXI1 & -0.683 & 0.002 & -0.511 & 0.03 \\
ORM1 & -0.594 & 0.009 & -0.670 & 0.002 \\
ZSCAN18 & & Ns & -0.511 & 0.030 \\
PXDN & -0.480 & 0.44 & -0.579 & 0.012 \\
\hline
\end{tabular}

The 100 most significant hypomethylated gene promoter sites were correlated to their respective gene expression. Only statistically significant correlations are shown $(p<0.05)$ 
Table 5 Correlation between the most differentially regulated genes and their promotor methylation

\begin{tabular}{lcccc}
\hline $\begin{array}{l}\text { Gene promoter/ } \\
\text { gene }\end{array}$ & $\begin{array}{l}\text { Pearson } \\
\text { coefficient }\end{array}$ & $P$ value & Spearman's rho & $P$-value \\
\hline SPP1 & ns & -0.523 & 0.026 \\
ALDH3A1 & ns & -0.560 & 0.016 \\
TCN1 & ns & -0.521 & 0.027 \\
\hline
\end{tabular}

The 130 most differentially regulated genes imported from our previous paper [17] were compared to corresponding gene promoter methylation levels. Significant correlations are shown $(p<0.05)$

the right of the heat map in Fig. 3 may illustrate a separate methylation profile within this subtype, which could be more prominent in a larger study. Different DNA methylation profiles in the histological subtypes have been suggested by Wang et al. who demonstrated global hypomethylation intestinal type cancers, and gene promoter hypermethylation in diffuse type cancers [23]. This paradox may explain why several other authors in the past have reported both genome wide hypermethylation and hypomethylation in GC tissue.

\section{Correlation with clinicopathological parameters and gene expression}

Further, we observed that DNA methylation of the GFRA3 gene promoter showed significant correlation with almost all clinicopathological parameters. Most importantly, high methylation levels of GFRA3 conferred a very unfavorable prognosis, with no high-expressing individuals surviving 20 months. GFRA3 codes for the artemin receptor which mediates activation of the RET proto-oncogene, and has been implicated in a GC diagnostic and prognostic signature [24]. In breast cancer, increased expression of GFRA3 was associated with lymph node metastasis and advanced tumor stage [32]. In pancreatic cancer GFRA3 may be implicated in the promotion of the disease through increased cell motility and invasiveness [33, 34], and this gene is also up regulated in non-small cell lung cancer [35]. We expected to see down regulation of the GFRA3 gene in the hypermethylated tumor specimens, however there was no

Table 6 KEGG cellular signaling pathways

\begin{tabular}{lcc}
\hline Pathway name & Impact factor & $p$-value \\
\hline Natural killer cell mediated cytotoxicity & 6.7 & 0.008 \\
Hematopoietic cell lineage & 5.1 & 0.017 \\
Colorectal cancer & 5.0 & 0.018 \\
Cytokine-cytokine receptor interaction & 5.4 & 0.022 \\
Bladder cancer & 4.8 & 0.029 \\
Endometrial cancer & 4.9 & 0.046
\end{tabular}

Significant associations between the 100 most hypomethylated CpG sites in the tumor relative to normal tissue, and KEGG cellular signal pathways (FDR corrected $p<0.05$ ). The 100 most hypermethylated CpG sites did not significantly associate with any KEGG pathways significant association between the GFRA3 methylation levels and gene expression in the study. Furthermore, it is widely accepted that there are distinct differences in the tumor biology between diffuse and intestinal GC. This difference, however, was not reflected in the GFRA3 promoter methylation levels, as we found no differences in GFRA3 methylation levels between the two histological types. GFRA3 may play different roles in the two cancer types, however a larger study is necessary to clarify whether there exists a true difference between the methylation of this gene in the two histological types and its significance.

In our previous study [17], we found that CLDN1 gene expression was highly associated with reduced postoperative survival, and that $I L-8$ was the most highly upregulated gene in the tumor specimens. Although there was no association between the methylation levels of these genes and their expression, we wanted to investigate the relationship between the most prominent findings in our two studies: $C L D N 1$ and $I L-8$ gene expression and GFRA3 promotor methylation: Indeed, the expression of $C L D N 1$ was statistically associated with both the $I L-8$ gene expression and GFRA3 promotor methylation. The association between GFRA3, IL-8 and CLDN1 and the clinical features such as post-operative survival, lymph node metastasis, gastric metaplasia and patient age may describe a more complex relationship which we have not further evaluated in this study.

Between the 200 most differentially methylated CpG sites, and the 130 most differentially regulated genes, there was a significant inverse relationship between methylation and gene expression in 13 of the genes, confirming that DNA methylation may be one of several regulatory mechanisms of gene expression in GC. However, other mechanisms than DNA methylation must account for the majority of gene regulation, such as gene mutations, and other epigenetic mechanisms like histone modifications, nucleosome positioning, noncoding RNAs, and microRNAs.

The cause of hypermethylation in gastric cancer is unclear, nonetheless infectious agents may be an important contributing factor. There is a strong association between $H$. pylori and GC, recognized by the World Health Organization as a class 1 carcinogen [36]. H. pylori triggers the chronic inflammatory process that results in the mucosal transformation leading to $\mathrm{GC}$, described by Correa [37] and later refined by Tahara [38]. The role of $H$. pylori in the methylation of gastric mucosal DNA, however, has not been extensively studied. It has been observed that $\mathrm{cag}^{+} H$. pylori infection results in both global hypomethylation in gastric mucosa [14-16] and hypermethylation of promoter regions of several tumor suppressor genes [39]. Chronic inflammation per se has also been shown to cause aberrant methylation in gastric 
epithelial cells [40]. Other infectious agents, such as the Epstein-Barr virus, have also been implicated in the promotion of DNA methylation in a subgroup of GC [39]. To evaluate the presence of $H$. pylori has not been the aim of this study. Up-regulation of the $I L-8$ gene is a general inflammatory marker and is also associated with H. pylori-induced inflammation. The strong association between expression of CLDN1 gene, methylation of GFRA3 promoter and expression of $I L-8$ gene in tumor tissue warrants further investigation in regards to the influence of $H$. pylori infection, but this is beyond the scope of this study.

Our main findings, such as the hypermethylated GFRA3 gene promoter and its possible prognostic role, should be further studied in a larger cohort of patients in the future.

\section{Conclusion}

In the present study, we demonstrated hypermethylation of the GFRA3 promoter region in GC samples, and identified an inverse relationship between the degree of GFRA3 hypermethylation and post-operative survival. GFRA3 was also associated with CLDN1 gene expression, a potential prognostic factor demonstrated in a previous study. The tumor and normal samples showed distinct DNA methylation profiles, indicating that aberrant DNA methylation may be a distinct feature of GC. Between the most aberrantly methylated gene promoters and corresponding gene expression there was only modest correlation, demonstrating that mechanisms other than DNA methylation must account for many of the changes in gene expression which occur in this disease.

\section{Additional file}

Additional file 1: $\beta$ values for selected CpG-sites. (TXT $7 \mathrm{~kb}$ )

\section{Abbreviations \\ GC: gastric cancer; CpG: cytosine-phosphate-guanine nucleotide sequence; CIMP: CpG island methylation phenotype; CLDN1: claudin-1; IL-8: interleukin- 8; GFRA3: GDNF family receptor alpha 3; Cag: cytotoxin associated gene; PCR: polymerase chain reaction; KEGG: Kyoto Encyclopedia of Genes and Genomes; IF: impact factor.}

\section{Competing interests}

The authors declare that they have no competing interests.

\section{Authors' contributions}

LLE included patients, obtained surgical biopsies and wrote the main manuscript. LLE and YE performed DNA isolation. JK performed the DNA methylation assays. VNK financed the arrays for methylation. JT processed and normalized the methylation data. GPB performed the histological examination and immunohistochemistry. GB performed part of the biostatistics and coordinated the study along with LLE and IRKB. All authors read and approved the manuscript.

\section{Acknowledgements}

The University of Oslo financed the project. The Illumina Infinium

Methylation Assay was performed by the Institute for Cancer Research at the
Radium Hospital, Oslo University Hospital. Dr Armand Borovik, Prince of Wales Hospital, Sydney, Australia, provided valuable comments.

\section{Author details}

'Department of Clinical Molecular Biology and Laboratory Science (EpiGen), Akershus University Hospital, Division of Medicine, Lørenskog, Norway. ${ }^{2}$ Department of Gastrointestinal Surgery, Akershus University Hospital, N-1478 Nordbyhagen, Lørenskog, Norway. ${ }^{3}$ K.G. Jebsen Center for Breast Cancer Research, Institute for Clinical Medicine, Faculty of Medicine, University of Oslo, Oslo, Norway. ${ }^{4}$ Department of Genetics, Institute for Cancer Research, OUS Radiumhospitalet Montebello, Oslo, Norway. ${ }^{5}$ Laboratory for Epigenetics and Environment, Centre National de Génotypage, CEA - Institut de Génomique, Evry, France. ${ }^{6}$ Department of Pathology, Akershus University Hospital, Lørenskog, Norway. Institute of Clinical Medicine, Akershus University Hospital and University of Oslo, Lørenskog, Norway. ${ }^{8}$ Infection Control and Environmental Health, Norwegian Institute of Public Health, Oslo, Norway. ${ }^{9}$ Department of Chemistry, Biotechnology and Food Science, Norwegian University of Life Sciences, Ås, Norway.

Received: 23 August 2015 Accepted: 2 March 2016

Published online: 16 March 2016

\section{References}

1. Lambert MP, Herceg Z. Epigenetics and cancer, 2nd IARC meeting, Lyon, France, 6 and 7 December 2007. Mol Oncol. 2008;2:33-40.

2. Sapari NS, Loh M, Vaithilingam A, Soong R. Clinical potential of DNA methylation in gastric cancer: a meta-analysis. PLoS One. 2012;7:e36275.

3. Ehrlich M. DNA hypomethylation in cancer cells. Epigenomics. 2009;1:239-59.

4. Ehrlich M, Lacey M. DNA hypomethylation and hemimethylation in cancer. Adv Exp Med Biol. 2013;754:31-56.

5. Jackson K, Yu MC, Arakawa K, Fiala E, Youn B, Fiegl H, et al. DNA hypomethylation is prevalent even in low-grade breast cancers. Cancer Biol Ther. 2004:3:1225-31.

6. Jones PA, Baylin SB. The fundamental role of epigenetic events in cancer. Nat Rev Genet. 2002:3:415-28.

7. Toyota M, Ahuja N, Ohe-Toyota M, Herman JG, Baylin SB, Issa JP. CpG island methylator phenotype in colorectal cancer. Proc Natl Acad Sci U S A. 1999;96:8681-6.

8. Christensen BC, Kelsey KT, Zheng S, Houseman EA, Marsit CJ, Wrensch MR et al. Breast cancer DNA methylation profiles are associated with tumor size and alcohol and folate intake. PLoS Genet. 2010;6:e1001043.

9. Christensen BC, Marsit CJ, Houseman EA, Godleski JJ, Longacker JL, Zheng S, et al. Differentiation of lung adenocarcinoma, pleural mesothelioma, and nonmalignant pulmonary tissues using DNA methylation profiles. Cancer Res. 2009;69:6315-21.

10. Christensen BC, Smith AA, Zheng S, Koestler DC, Houseman EA, Marsit CJ, et al. DNA methylation, isocitrate dehydrogenase mutation, and survival in glioma. J Natl Cancer Inst. 2011;103:143-53.

11. Zong L, Seto Y. CpG island methylator phenotype, Helicobacter pylori, Epstein-Barr virus, and microsatellite instability and prognosis in gastric cancer: a systematic review and meta-analysis. PLoS One. 2014;9:e86097.

12. Suzuki H, Toyota M, Sato H, Sonoda T, Sakauchi F, Mori M. Roles and causes of abnormal DNA methylation in gastrointestinal cancers. Asian Pac J Cancer Prev. 2006;7:177-85.

13. Sitaraman R. Helicobacter pylori DNA methyltransferases and the epigenetic field effect in cancerization. Front Microbiol. 2014;5:115

14. Compare D, Rocco A, Liguori E, D'Armiento FP, Persico G, Masone S, et al. Global DNA hypomethylation is an early event in Helicobacter pylori-related gastric carcinogenesis. J Clin Pathol. 2011;64:677-82.

15. Shin CM, Kim N, Jung Y, Park JH, Kang GH, Park WY, et al. Genome-wide DNA methylation profiles in noncancerous gastric mucosae with regard to Helicobacter pylori infection and the presence of gastric cancer. Helicobacter. 2011;16:179-88.

16. Yoshida T, Yamashita S, Takamura-Enya T, Niwa T, Ando T, Enomoto S, et al. Alu and Satalpha hypomethylation in Helicobacter pylori-infected gastric mucosae. Int J Cancer. 2011;128:33-9.

17. Eftang LL, Esbensen Y, Tannaes TM, Blom GP, Bukholm IR, Bukholm G. Upregulation of CLDN1 in gastric cancer is correlated with reduced survival. BMC Cancer. 2013;13:586. 
18. Eftang LL, Esbensen Y, Tannaes TM, Bukholm IR, Bukholm G. Interleukin-8 is the single most up-regulated gene in whole genome profiling of $\mathrm{H}$. pylori exposed gastric epithelial cells. BMC Microbiol. 2012;12:9.

19. Ding Y, Le XP, Zhang QX, Du P. Methylation and mutation analysis of p16 gene in gastric cancer. World J Gastroenterol. 2003;9:423-6.

20. Fang DC, Wang RQ, Yang SM, Yang JM, Liu HF, Peng GY, et al. Mutation and methylation of $\mathrm{hMLH1}$ in gastric carcinomas with microsatellite instability. World J Gastroenterol. 2003;9:655-9.

21. Machado JC, Oliveira C, Carvalho R, Soares P, Berx G, Caldas C, et al. Ecadherin gene $(\mathrm{CDH} 1)$ promoter methylation as the second hit in sporadic diffuse gastric carcinoma. Oncogene. 2001;20:1525-8.

22. Nojima M, Suzuki H, Toyota M, Watanabe Y, Maruyama R, Sasaki S, et al. Frequent epigenetic inactivation of SFRP genes and constitutive activation of Wnt signaling in gastric cancer. Oncogene. 2007;26:4699-713.

23. Wang K, Yuen ST, Xu J, Lee SP, Yan HH, Shi ST, et al. Whole-genome sequencing and comprehensive molecular profiling identify new driver mutations in gastric cancer. Nat Genet. 2014;46:573-82.

24. Cui J, Li F, Wang G, Fang X, Puett JD, Xu Y. Gene-expression signatures can distinguish gastric cancer grades and stages. PLoS One. 2011;6:e17819.

25. Ehrlich M, Gama-Sosa MA, Huang LH, Midgett RM, Kuo KC, McCune RA, et al. Amount and distribution of 5-methylcytosine in human DNA from different types of tissues of cells. Nucleic Acids Res. 1982:10:2709-21.

26. Guan Z, Zhang J, Song S, Dai D. Promoter methylation and expression of TIMP3 gene in gastric cancer. Diagn Pathol. 2013:8:110.

27. Chen R, Zhuge X, Huang Z, Lu D, Ye X, Chen C, et al. Analysis of SEMA3B methylation and expression patterns in gastric cancer tissue and cell lines. Oncol Rep. 2014;31:1211-8.

28. Li H, Wang J, Xu H, Xing R, Pan Y, Li W, et al. Decreased fructose-1,6bisphosphatase-2 expression promotes glycolysis and growth in gastric cancer cells. Mol Cancer. 2013;12:110.

29. Lim B, Park JL, Kim HJ, Park YK, Kim JH, Sohn HA, et al. Integrative genomics analysis reveals the multilevel dysregulation and oncogenic characteristics of TEAD4 in gastric cancer. Carcinogenesis. 2014;35:1020-7.

30. Miao R, Guo X, Zhi Q, Shi Y, Li L, Mao X, et al. VEZT, a novel putative tumor suppressor, suppresses the growth and tumorigenicity of gastric cancer. PLoS One. 2013;8:e74409.

31. Asada K, Ando T, Niwa T, Nanjo S, Watanabe N, Okochi-Takada E, et al. FHL1 on chromosome $X$ is a single-hit gastrointestinal tumor-suppressor gene and contributes to the formation of an epigenetic field defect. Oncogene. 2013;32:2140-9.

32. Wu ZS, Pandey V, Wu WY, Ye S, Zhu T, Lobie PE. Prognostic significance of the expression of GFRalpha1, GFRalpha3 and syndecan-3, proteins binding ARTEMIN, in mammary carcinoma. BMC Cancer. 2013;13:34.

33. Meng LX, Chi YH, Wang XX, Ding ZJ, Fei LC, Zhang H, et al. Neurotrophic artemin promotes motility and invasiveness of MIA PaCa-2 pancreatic cancer cells. Asian Pac J Cancer Prev. 2012;13:1793-7.

34. Zhu DL, Luo DL, Luo G, Wang B, Gao JM. Artemin and GFRalpha3 expressions and their relevance to perineural invasiveness and metastasis of pancreatic carcinoma. Nan Fang Yi Ke Da Xue Xue Bao. 2009;29:428-32.

35. Tang JZ, Kong XJ, Kang J, Fielder GC, Steiner M, Perry JK, et al. Arteminstimulated progression of human non-small cell lung carcinoma is mediated by BCL2. Mol Cancer Ther. 2010;9:1697-708.

36. Schistosomes, liver flukes and Helicobacter pylori. IARC Working Group on the Evaluation of Carcinogenic Risks to Humans. Lyon, 7-14 June 1994. IARC Monogr Eval Carcinog Risks Hum 1994, 61: 1-241.

37. Correa P, Haenszel W, Cuello C, Tannenbaum S, Archer M. A model for gastric cancer epidemiology. Lancet. 1975;2:58-60

38. Tahara E. Genetic pathways of two types of gastric cancer. IARC Sci Publ. 2004; 327-349

39. Matsusaka K, Funata S, Fukayama M, Kaneda A. DNA methylation in gastric cancer, related to Helicobacter pylori and Epstein-Barr virus. World J Gastroenterol. 2014;20:3916-26.

40. Niwa T, Tsukamoto T, Toyoda T, Mori A, Tanaka H, Maekita T, et al. Inflammatory processes triggered by Helicobacter pylori infection cause aberrant DNA methylation in gastric epithelial cells. Cancer Res. 2010;70:1430-40.

\section{Submit your next manuscript to BioMed Central and we will help you at every step:}

- We accept pre-submission inquiries

- Our selector tool helps you to find the most relevant journal

- We provide round the clock customer support

- Convenient online submission

- Thorough peer review

- Inclusion in PubMed and all major indexing services

- Maximum visibility for your research

Submit your manuscript at www.biomedcentral.com/submit

) Biomed Central 